\begin{tabular}{cc}
\hline & International Journal of Engineering \& Technology, $7(4.5)(2018) 560-565$ \\
SPC & Website: $\frac{w w w . s c i e n c e p u b c o . c o m / i n d e x . p h p / I J E T}{2}$ \\
Research paper & Technology \\
\hline
\end{tabular}

\title{
Health care monitoring system an application of IOT using WI-FI
}

\author{
Kusum Grewal Dangi ${ }^{1}$, Mohit Yadav $^{1}$, Shubham Malhotra ${ }^{1}$ \\ ${ }^{1}$ EECE Dept., The North Cap University \\ *Corresponding author E-mail: kusumgrewal@ncuindia.edu
}

\begin{abstract}
With emerging networking technologies and micro electromechanical devices the world is full of opportunities to get connected and stay connected seamlessly. To facilitate this connectivity among every possible object in the world, Internet of things (IoT) has emerged. In this project we have made a hardware consists of a Micro-Controller, Pulse Measurement Sensor, and Body Temperature Sensor, Wi-Fi module, LCD and some interfacing module. So basically this hardware will take reading from the sensors and after processing that it will show the readings/measurement on LCD as well as on a server which is online. The major thing in this project is that one can monitor the patient at anywhere in the world with the use of internet, the doctor keep the track of your health and measurement of heart rate, blood pressure, etc. In this way you always be in touch with the Doctor, no matter where you are. Then if something happen apparently the message goes to the doctor before it becomes the emergency situation and also if a patient is hospitalized then patients would also be allowed to leave hospitals and clinics earlier, as professionals are enabled to monitor them from home.
\end{abstract}

Keywords: IOT; Medical \& Healthcare; Wi-Fi; Connectivity; ICT.

\section{Introduction}

Internet of things (IoT) is the network of network of things. With this concept of IoT, we will be able to connect not only the existing network devices but also all the things around the world. For an instance remote controlling our home appliances with the help of local area network technologies. It leads to a world where the real, digital and the virtual are converging to create smart envi- ronment. This technique uses the concept of various network technologies like wide area network, local area network and per- sonal area network to connect the objects at various ranges, along with the concept of cloud computing and big data.

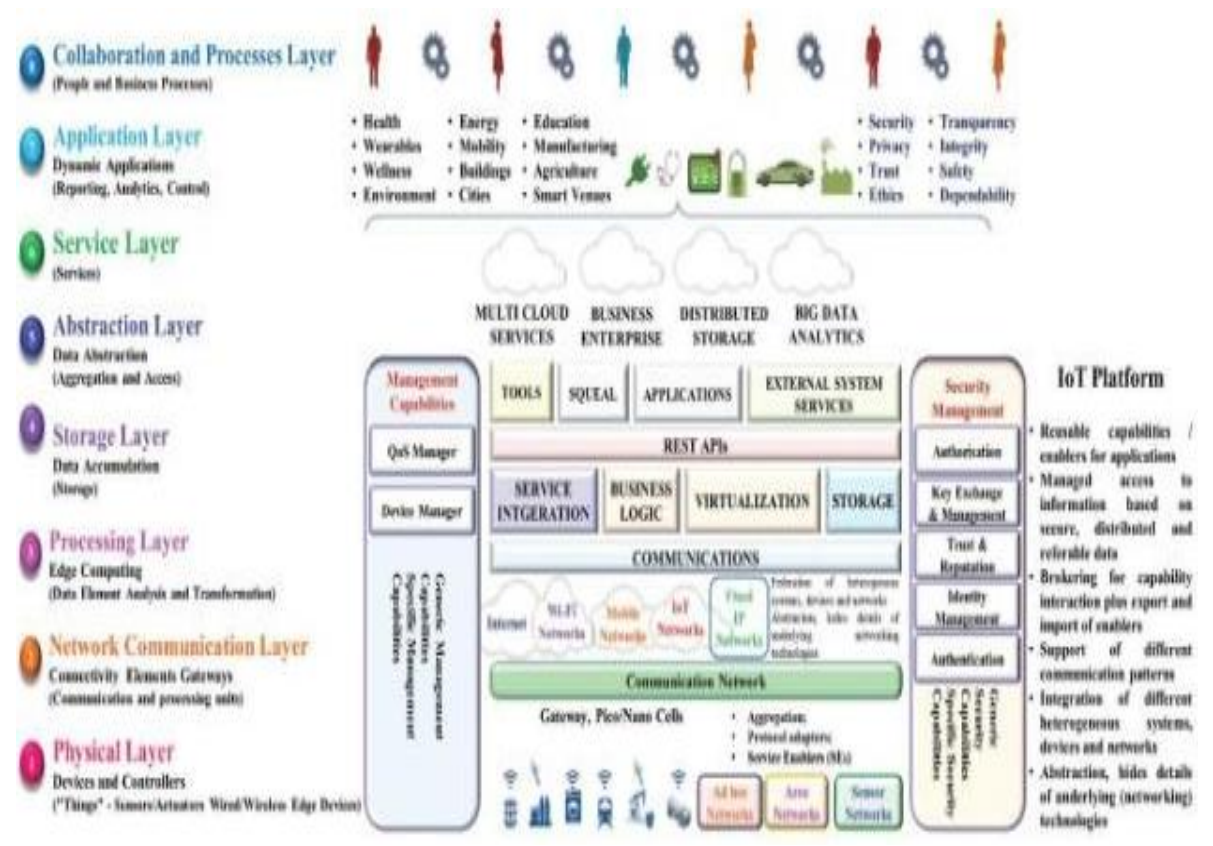

Fig. 1: IOT Architecture [1]. 


\section{IOT application}

\subsection{Smart}

1) City: With the use of Internet of things, future cities will be greener, safer and more efficient. As with the help of IoT everything can be connected such as your home appliances, car, etc. with internet and you can control or monitor them from everywhere. In future everything will be automatic in smart cities, many different stakeholders must work together to provide the best technology solutions. Network operators, managed service providers, system integrators and technol- ogy providers all have a role to play in working with gov- ernments to enable smart city solutions [5].

2) Homes: Future home will become more and more automated because of Internet of things. All devices and appliances in home will be connected through your phone so that you can monitor them [5]. They will become self-controlled. As we all know now a days everything is wireless, wireless communication is very efficient and if we use this technology along with IoT in home then the home would be called the smart homes. For example if your refrigerator is short of milk then your refrigerator will ping you that the I am short of milk, kindly bring the milk, It's very fascinating. This is how the IoT works.

3) Grid: Energy consumption is one the serious issue these days With the use IoT, enables efficient resource utilisation to optimize the energy consumption. The Smart grid is the combination of traditional energy power grid with the telecommunication and information technology. Such integration enables efficient resource utilization to optimize energy consumption, install and manage distributed energy sources, as well as to exchange the generated power. In other words, the power flow and communications will be in two-ways.

4) Retail: As online retailing has already ruling the market now a days. The Internet of Things presents an opportunity for retailers to develop a vastly improved ecosystem that connects physical and digital worlds, allowing bidirectional, real-time interaction with consumers both inside and outside the store. The future retailing will be like browse products in-retail store and then check out the rates at different stores and after that order it online wherever you find that product cheaper in rate. This is the one kind of e-retailing. In fact some of brands like Hudson's Bay and Lord \& Taylor's have already started this kind of retailing. In South Korea this technology has already implemented [10]

5) Optimization of Supply chain Operations: The budding applications of Internet of Things (IoT) technology across any industry or sector are vast. Prevailing and future application of IoT gives assurance to new ways of value creation and revenue streams for business in a digital world. For supply chain operations IoT technologies allow creation of a broad framework within which rapid evolution in an increasingly complex environment can be visualized and implemented. This evolution is based on the possibility of being able to in- tegrate all relevant information in any value chain, under- stand the interdependencies amongst those pieces of informa- tion almost instantaneously, and leverage advanced analytics to arrive at more optimized decisions. An IoT enables the supply chain operations can be envisaged as a smart interre- lated network that predicaments composed multiple tiers of suppliers, contract manufacturers, service providers, distribu- tors and customers, physically located across different re- gions of the world. Information created, processed and inter- preted by distributed smart objects and systems in this intelli- gent network eliminates visibility gaps.

6) Farming: With the help of Internet of Things, the farmers would be able to monitors the essential conditions like weather, moisture of soil and amount of fertilizers required for the crops. In this manner they can grow more crops efficiently.

\subsection{Wearables}

These days wearable are very helpful as they monitors, blood pressure, heart rate, body temperature and many more things.

\subsection{Connectivity}

1) Car /Automobiles: As the number of connected vehicles continues to grow, automotive use cases can be grouped into five main categories: Infotainment: Voice communications, personalized music, Navigation: Traffic information, online route planning, Safety: Smart SOS (e-Call), roadside assistance, Cost-efficiency: Insurance telematics, remote diagnostics, and condition-based maintenance, Payment: Electronic toll collection, parking reservation and payment.

2) Health Care and Medical System: In medical health care IoT will play a very important role. In medical and healthcare IoT refers to any corporal entity or body embedded with a technology which is proficient in exchanging data and is attached or fixed in the health unit to create a more efficient healthcare system. This is one type of the example and another would be the doctor keep the track of your health and measurement of different quantity like heart rate, blood pressure, etc, you just need to wear a hand bracelet which is connected through internet that keeps recording your heart beat and other body measurement. In his way you always be in touch with the Doctor no matter where you are. Then if something happen apparently the message goes to the doctor before it becomes the emergency situation and also if a patient is hospitalized then patients would also be allowed to leave hospitals and clinics earlier, as professionals are enabled to monitor them from home rather than keeping them in hospitals for observation.

\section{General concept healthcare $\&$ medical unit}

In medical health care IoT will play a very important role. In medical and healthcare IoT refers to any corporal entity or body embedded with a technology which is proficient in exchanging data and is attached or fixed in the health unit to create a more efficient healthcare system. This is one type of the example and another would be the doctor keep the track of your health and measurement of different quantity like heart rate, blood pressure, etc, you just need to wear a hand bracelet which is connected through internet that keeps recording your heart beat and other body measurement. In his way you always be in touch with the Doctor no matter where you are [9]. Then if something happen apparently the message goes to the doctor before it becomes the emergency situation and also if a patient is hospitalized then pa- tients would also be allowed to leave hospitals and clinics earlier, as professionals are enabled to monitor them from home rather than keeping them in hospitals for observation

\subsection{Problem statement}

As in this project the doctor can monitor the patient from anywhere in the world through internet, which was not possible earlier because IoT came into existence recently. This is not applicable only to patients but also a well \& healthy person, as he or she can wear some device which continuously send the data to the healthcare unit. If something will happen wrong then immediately cure can be provided as the person is under the supervision of doctors $24 X 7$. With this emerging technology now the death due to health issue can be less as compared with the old days 


\section{Details of module used}

\subsection{Wi-Fi module}

ESP-12E WiFi module is developed by Ai-thinker Team. core processor ESP8266 in smaller sizes of the module encapsulates Tensilica L106 integrates industry-leading ultra low power 32-bit MCU micro, with the 16-bit short mode, Clock speed support $80 \mathrm{MHz}$, $160 \mathrm{MHz}$, supports the RTOS, integrated Wi-Fi $\mathrm{MAC} / \mathrm{BB} / \mathrm{RF} / \mathrm{PA} / \mathrm{LNA}$, on-board antenna. The module supports standard IEEE $802.11 \mathrm{~b} / \mathrm{g} / \mathrm{n}$ agreement, complete TCP/IP protocol stack. Users can use the add modules to an existing device networking, or building a separate network controller. ESP8266 is high integration wireless SOCs, designed for space and power constrained mobile platform designers. It provides unsurpassed ability to embed Wi-Fi capabilities within other systems, or to function as a standalone application, with the lowest cost, and minimal space requirement.

ESP8266EX offers a complete and self-contained Wi-Fi networking solution; it can be used to host the application or to offload WiFi networking functions from another application processor. When ESP8266EX hosts the application, it boots up directly from an external flash. In has integrated cache to improve the perform- ance of the system in such applications. Alternately, serving as a Wi-Fi adapter, wireless internet access can be added to any micro controller based design with simple connectivity (SPI/SDIO or I2C/UART interface) [15]. ESP8266EX is among the most inte- grated WiFi chip in the industry; it integrates the antenna switches, RF balun, power amplifier, low noise receive amplifier, filters, power management modules, it requires minimal external circuitry, and the entire solution, including front-end module, is designed to occupy minimal PCB area.

\subsection{Micro controller}

Microcontroller, as the name suggests, are small controllers. They are like single chip computers that are often embedded into other systems to function as processing/controlling unit. For example, the remote control you are using probably has microcontrollers inside that do decoding and other controlling functions. They are also used in automobiles, washing machines, microwave ovens, toys etc, where automation is needed [18]. Here we are using AVR AT Mega 328 microcontroller based on Arduino Platform. Platform refers to hardware architecture with software framework on which other software can run.

\subsection{Arduino}

Arduino is an open-source electronics prototyping platform based on flexible, easy-to-use hardware and software. It's intended for artists, designers, hobbyists, and anyone interested in creating interactive objects or environments. Arduino can sense the envi- ronment by receiving input from a variety of sensors and can af- fect its surroundings by controlling lights, motors, and other ac- tuators. The microcontroller on the board is programmed using the Arduino programming language (based on Wiring) and the Ardu- ino development environment (based on Processing). Arduino projects can be stand-alone or they can communicate with soft- ware running on a computer (e.g. Flash, Processing, MaxMSP).The boards can be built by hand or purchased preas- sembled; the software can be downloaded for free. The hardware reference designs (CAD files) are available under an open-source license, you are free to adapt them to your needs. Arduino re- ceived an Honorary Mention in the Digital Communities section of the 2006 Ars Electronica Prix. The Arduino team is: Massimo Banzi, David Cuartielles, Tom Igoe, Gianluca Martino, and David Mellis [19].

The Arduino Uno is a microcontroller board based on the ATmega328 (datasheet). It has 14 digital input/output pins (of which 6 can be used as PWM outputs), 6 analog inputs, a $16 \mathrm{MHz}$ ce- ramic resonator, a USB connection, a power jack, an ICSP header, and a reset button. It contains everything needed to support the microcontroller; simply connect it to a computer with a USB cable or power it with a AC-to-DC adapter or battery to get started. The Uno differs from all preceding boards in that it does not use the FTDI USB-toserial driver chip. Instead, it features the At- mega16U2 (Atmega8U2 up to version R2) programmed as a USB-to-serial converter. Revision 2 of the board has the following new features.

\subsection{LCD}

LCD (Liquid Crystal Display) screen is an electronic display module and find a wide range of applications. A 16×2 LCD dis-play is very basic module and is very commonly used in various devices and circuits. These modules are preferred over seven seg- ments and other multi segment LEDs. The reasons being: LCDs are economical; easily programmable; have no limitation of dis- playing special even custom characters (unlike in seven seg- m5 ents), animations and so on.

A 16x2 LCD means it can display 16 characters per line and there are 2 such lines. In this LCD each character is displayed in $5 \times 7$ pixel matrix. 16 Characters x 2 Lines Built-in HD44780 Equiva- lent LCD Controller Works directly with ATMEGA, ARDUINO, PIC ARM and 8051 many other microcontroller/kits.4 or 8 bit data I/O interface Low power consumption Datasheet available on the Internet. This LCD has two registers, namely, Command and Data. Command register is used to insert a special command into the LCD [16]. While data register is used to insert a data into the LCD. Command is a special set of data which is used to give in- ternal command to LCD. Like clear screen, move to line 1character, setting up the cursor etc.

\subsection{Easy heart rate sensor}

The new version uses the TCRT1000 reflective optical sensor for photo plethysmography. The use of TCRT100 simplifies the build process of the sensor part of the project as both the infrared light emitter diode and the detector are arranged side by side in a leaded package, thus blocking the surrounding ambient light, which could otherwise affect the sensor performance. I have also designed a printed circuit board for it, which carries both sensor and signal conditioning unit. and its output is a digital pulse which is synchronous with the heart beat. The output pulse can be fed to either an ADC channel or a digital input pin of a microcontroller for further processing and retrieving the heart rate in beats per minute (BPM) [9]. The sensor used in this project is TCRT1000, which is a reflective optical sensor with both the infrared light emitter and phototransistor placed side by side and are enclosed inside a leaded package so that there is minimum effect of surrounding visible light. The circuit diagram below shows the external biasing circuit for the TCRT1000 sensor. Pulling the Enable pin high will turn the IR emitter LED on and activate the sensor. A fingertip placed over the sensor will act as a reflector of the incident light. The amount of light reflected back from the fingertip is monitored by the phototransistor.

The output (VSENSOR) from the sensor is a periodic physiological waveform attributed to small variations in the reflected IR light which is caused by the pulsatile tissue blood volume inside the finger. The waveform is, therefore, synchronous with the heart beat. The following circuit diagram describes the first stage of the signal conditioning which will suppress the large DC component and boost the weak pulsatile $\mathrm{AC}$ component, which carries the required information.

In the circuit shown above, the sensor output is first passed through a RC high-pass filter (HPF) to get rid of the DC compo- nent. The cut-off frequency of the HPF is set to $0.7 \mathrm{~Hz}$. Next stage is an active low-pass filter (LPF) that is made of an Op-Amp cir- cuit. The gain and the cut-off frequency of the LPF are set to 101 and $2.34 \mathrm{~Hz}$, respectively. Thus the combination of the HPF and LPF helps to remove unwanted DC signal and high frequency noise including 60 $\mathrm{Hz}(50 \mathrm{~Hz}$ in some countries) mains interfer- ence, while amplifying the low amplitude pulse signal (AC com- ponent) 101 times. 
The output from the first signal conditioning stage goes to a similar HPF/LPF combination for further filtering and amplification (shown below). So, the total voltage gain achieved from the two cascaded stages is $101 * 101=10201$. The two stages of filtering and amplification converts the input PPG signals to near TTL pulses and they are synchronous with the heart beat. The fre quency (f) of these pulses is related to the heart rate (BPM) as, Beats per minute

$(\mathrm{BPM})=60 * \mathrm{f}$.

\subsection{Temperature sensor}

LM35 is a precision IC temperature sensor with its output proportional to the temperature (in oC). The sensor circuitry is sealed and therefore it is not subjected to oxidation and other processes. With LM35, temperature can be measured more accurately than with a thermistor. It also possess low self heating and does not cause more than $0.1 \mathrm{oC}$ temperature rise in still air. The operating tem- perature range is from $-55^{\circ} \mathrm{C}$ to $150^{\circ} \mathrm{C}$. The output voltage varies by $10 \mathrm{mV}$ in response to every oC rise/fall in ambient temperature, i.e., its scale factor is $0.01 \mathrm{~V} / \mathrm{oC}$.

\section{Block diagram of project}

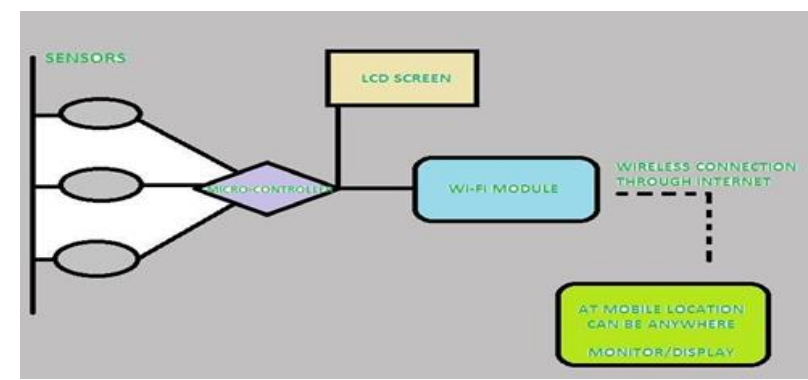

Fig. 2: Block Diagram.

\subsection{Description}

We have used a $12 \mathrm{~V}$ power supply (adapter), which supplies power to the circuit. As the limit of the components of our circuit is Max of $5.5 \mathrm{~V}$, we have used a buck converter, which is represented in the circuit as LM2596 Module, which is a step down converter. It is a DC-DC power convertor, which brings down the voltage, while stepping up the current, thus protecting the components from getting damaged. We have also used a LED, which can be seen next to the LM 2596. The LED glows, when the signals trav- el from sensor to the microcontroller Atmega 328, which process- es it and sends it to the $16 \times 2 \mathrm{LCD}$. The information is displayed on the LCD. There is small potentiometer next to the LCD dis- play, which controls the contrast of the pixels in the LCD. The signals then travel to the 4Channel, Bidirectional Logic Level Shifter, whose main work is to bring down the signal from $5 \mathrm{v}$ to

$3.3 \mathrm{v}$ so that it can be further fed to Wi-Fi chip - ESP8266, which is responsible for sending data to the server, so that it can be ana- lysed and get displayed. Along with the Wi-Fi chip we have used LM 1117 Module. The temperature senor used is LM-35, which sends the signals to microcontroller in analog form.

\subsection{Realistic view of project}

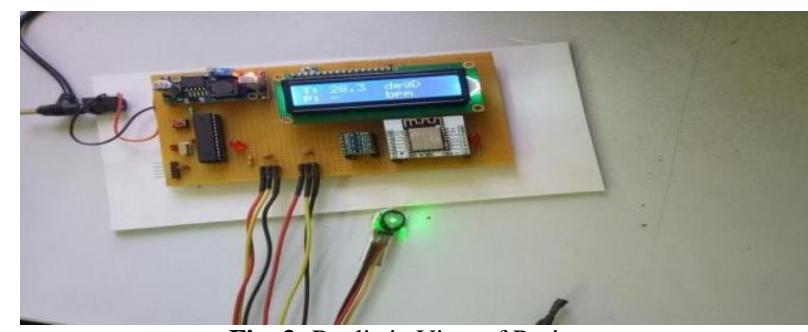

Fig. 3: Realistic View of Project.

\section{Programming environment}

\subsection{Arduino}

Arduino is an open-source electronics prototyping platform based on flexible, easy-to-use hardware and software. It's intended for artists, designers, hobbyists, and anyone interested in creating interactive objects or environments. Arduino can sense the envi- ronment by receiving input from a variety of sensors and can af- fect its surroundings by controlling lights, motors, and other actua- tors. The microcontroller on the board is programmed using the Arduino programming language (based on Wiring) and the Arduino development environment (based on Processing). Arduino projects can be stand-alone or they can communicate with software running on a computer (e.g. Flash, Processing, MaxMSP).The boards can be built by hand or purchased preas- sembled [25]; the software can be downloaded for free. The hardware reference designs (CAD files) are available under an open-source license, you are free to adapt them to your needs. Arduino received an Honorary Mention in the Digital Communi- ties section of the 2006 Ars Electronica Prix. The Arduino team is: Massimo Banzi, David Cuartielles, Tom Igoe, Gianluca Marti- no, and David Mellis.

\subsection{Thing speak open source free server}

Thing Speak is a platform that lets the user, store and analyse the data, recorded from sensors. It is an IOT platform. In this project we have used Arduino to send the data to Thing Speak. We have been given a login account for thing speak, and an API channel key.

With this key Thing Speak is able to detect, that the data has been sent from our channel and needs to be displayed on my account window. We can send data to thing Speak from other hardwares also, i.e - Raspberry pi, Beagle Bone Black.

\section{Existing features of IOT}

1) Real Time Data Analytics: Real-time data analytics is the use of data and related resources as soon as the data enters the system. The adjective real-time refers to a level of com- puter openness that a user senses as immediate or nearly in- stantaneous. Real-time analytics is also known as active scru- tiny, real-time analysis, real-time data integration and real- time intellect [15].

2) Benchmarks: The IoT software platforms need to be scalable and should incorporate amenities to describe and assess the system performance. Well demarcated performance metrics need to be planned to prototypical and measure the performance of IoT structures, taking into account network characteristics, energy feasting physiognomies, system throughput, computational resource consumption, and other effective features [7].

3) Edge Analytics: Measures need to be taken to diminish the huge network bandwidth ingesting conceivable between the sensorand the IoT server. Use of frivolous communication protocols is one key solution. And other approach is edge analytics, which can reduce the amount of raw data transmitted to the IoT server. This could be executed even in simple hardware embedded systems, such as an Arduino [7].

4) Device Management and Integration Support: Device supervision is one of the most significant feature in IoT software platform. The IoT platform can maintain a list of devices linked to it and track their action status; it should be able to handle configuration, firmware apprises and offer device level error reporting and error handling [14].

Companies tolerate is having an unwavering universal dignity or protocol for all of this extra data that is being collected 


\section{Result}

\subsection{Readings}

We can take the readings from both LCD \& Online Server anywhere in the world.

Table 1: Readings of Pulse and Temperature

\begin{tabular}{|c|c|c|c|}
\hline Created at (Time) & Entry Id & Pulse Rate & Temperature \\
\hline \multicolumn{4}{|l|}{$2017-05-13$} \\
\hline 10:07:21 UTC & 1 & 0 & 27 \\
\hline \multicolumn{4}{|l|}{$2017-05-13$} \\
\hline 10:07:51 UTC & 2 & 116 & 28 \\
\hline \multicolumn{4}{|l|}{$2017-05-13$} \\
\hline 10:08:21 UTC & 3 & 94 & 28 \\
\hline \multicolumn{4}{|l|}{$2017-05-13$} \\
\hline 10:08:51 UTC & 4 & 82 & 28 \\
\hline \multicolumn{4}{|l|}{$2017-05-13$} \\
\hline 10:09:21 UTC & 5 & 93 & 27 \\
\hline \multicolumn{4}{|l|}{$2017-05-13$} \\
\hline 10:09:51 UTC & 6 & 80 & 28 \\
\hline \multicolumn{4}{|l|}{$2017-05-13$} \\
\hline 10:10:21 UTC & 7 & 78 & 27 \\
\hline \multicolumn{4}{|l|}{$2017-05-13$} \\
\hline 10:10:51 UTC & 8 & 79 & 27 \\
\hline \multicolumn{4}{|l|}{$2017-05-13$} \\
\hline $10: 11: 21$ UTC & 9 & 85 & 35 \\
\hline \multicolumn{4}{|l|}{$2017-05-13$} \\
\hline 10:11:51 UTC & 10 & 0 & 30 \\
\hline \multicolumn{4}{|l|}{$2017-05-13$} \\
\hline $10: 12: 22$ UTC & 11 & 0 & 31 \\
\hline \multicolumn{4}{|l|}{$2017-05-13$} \\
\hline 10:12:51 UTC & 12 & 0 & 31 \\
\hline \multicolumn{4}{|l|}{$2017-05-13$} \\
\hline $10: 13: 21$ UTC & 13 & 0 & 30 \\
\hline \multicolumn{4}{|l|}{$2017-05-13$} \\
\hline 10:13:51 UTC & 14 & 69 & 28 \\
\hline \multicolumn{4}{|l|}{$2017-05-13$} \\
\hline $10: 14: 21$ UTC & 15 & 132 & 28 \\
\hline \multicolumn{4}{|l|}{$2017-05-13$} \\
\hline 10:14:51 UTC & 16 & 0 & 27 \\
\hline \multicolumn{4}{|l|}{$2017-05-13$} \\
\hline $10: 15: 21$ UTC & 17 & 0 & 45 \\
\hline \multicolumn{4}{|l|}{$2017-05-13$} \\
\hline 10:15:51 UTC & 18 & 99 & 34 \\
\hline \multicolumn{4}{|l|}{$2017-05-13$} \\
\hline 10:56:36 UTC & 19 & 76 & 28 \\
\hline
\end{tabular}

\subsection{Readings on online server think speak}

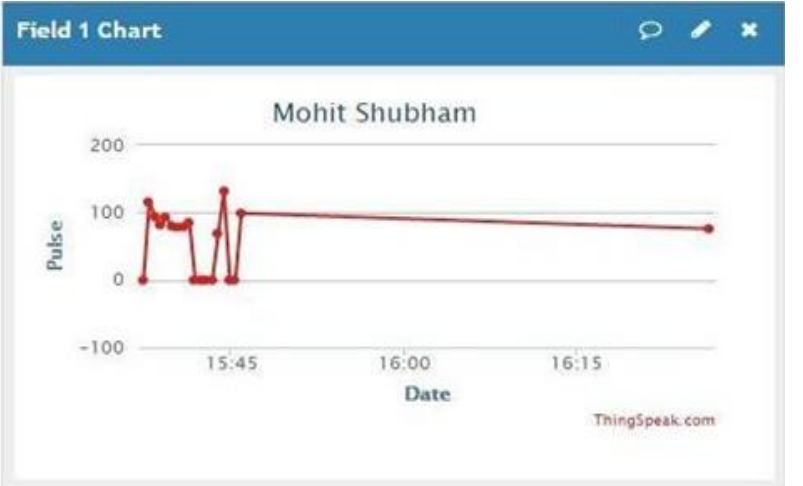

Fig. 4: Pulse Reading.

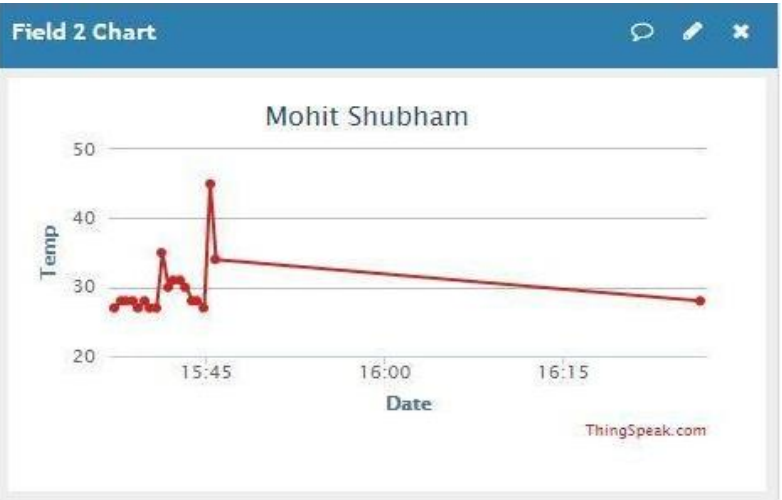

Fig. 5: Temperature Reading.

\section{Conclusion \& future scope}

The technology used in the project is one of the few ways of monitoring patients health. We have used IOT, which as of now seems to be providing a solution to every human reality can be little expensive. We could have also used Zig-Bee instead of Wi- Fi, to transfer the data, to the server. Zig-Bee holds an edge over Wi-Fi and Bluetooth, i.e (provides larger bandwidth and data rate). But using Zig-Bee, is not feasible due to high cost.

In future we can use this technology not only in monitoring temperature, pulse, small measurement but also we can monitor the ECG, EMG, EKG, Blood Pressure, Breath Rate, etc. from anywhere in the world. So whenever something goes wrong then immediately cure can be provided. The future work is to include a ZigBee module in this project so that in ICU readings of multiple patients can be monitored from any location. As we can connect as many nodes we want to connect with Zig-Bee. Which can further received by the Wi-Fi Module \& can be monitored from anywhere online through internet.

\section{Acknowledgement}

We express our deep sense of gratitude to Mrs. Kusum Grewal Dangi (Asst. Professor Sr. Scale), Department of Electrical, Electronics \& Communication Engineering, The NorthCap University (Gurugram), for her invaluable guidance, persistent encouragement and constructive supervision during the course of my study. We are indebted to her for sparing her valuable time in giving me suggestions and increasing my knowledge through fruitful discussions. We express our deep appreciation and sincere thanks to Dr. Swaran Ahuja, Head of the Department Electrical, Electronics \& Communication Engineering for providing all kinds of possible help and encouragement during my project. We owe thanks to library staff, Lab Technicians of The NorthCap University (Gurugram) for their full co-operation in providing the necessary literature \& helping. We will be failing in my duties, if we don't acknowledge my sense of gratitude to God Almighty and my parents, the blessings of whom have made me reach my destination.

\section{References}

[1] Vermesan, O. et al, Internet of Things beyond the Hype: Research, Innovation and Deployment, IERC Cluster SRIA 2015, http://www.internet-of-things-research.eu/pdf/Internet of Things beyond the Hype - Chapter 3 - SRIA - IERC 2015_Cluster eBook 978-87- 93237-98-8_P_Web.pdf.

[2] Internet Link, https://iot-analytics.com/10-internet-of-things- applications/ on 20 January 2017.

[3] Internet link, "https://dzone.com/articles/iot-software-platformcomparison".

[4] Cyber Security [K. Yoshigoe, W. Dai, M. Abramson and A. Ja- cobs, "Overcoming invasion of privacy in smart home environ- ment with synthetic packet, Injection, TRON Symposium (Tronshow) 2015 Tokyo Japan, 2014 pp 1-7, doi 10.1109/TRONSHOW.2014.7396875]. 
[5] Andrea Zanella, Nicola Bui, Angelo P. Castellani, Lorenzo Van- gelista , Michele Zorzi, “Internet of Things for Smart Cities", IEEE Internet of things Journal, Volume: 1, Issue: 1, Feb. 2014.

[6] Shanzhi chen, Hui Xu, Dake Liu, Bo Hu, Huncheng Wang, "A Vision of IoT: Applications, Challenges, and Opportunities With China Perspective" IEEE Internet of things Journal, Voume: 1, is- sue 4, Aug. 2014.

[7] J. A. Stankovic, "Research directions for the Internet of Things", IEEE Internet Things J., vol. 1, no. 1, pp. 3-9, Feb. 2014.

[8] Jan Henrik Ziegeldorf1*, Oscar Garcia Morchon2, and Klaus Wehrle1, "Privacy in the Internet of Things: Threats and Challenges" SECURITY AND COMMUNICATION NETWORKS Security Comm. Networks 2013 DOI: 10.1002/sec.795.

[9] Ahmed W. Atamli, Andrew Martin, "Threat-Based Security Analysis for the Internet of Things", 2014 International Workshop on Secure Internet of Things, DOI: 10.1109/SIoT.2014.10, INSPEC Accession Number: 1498511.

[10] Internet link,

[11] "https://www.softwareag.com/corporate/images/SAG_Connected_ Retail_Planet_Retail_8PG_WP_Mar16-Web_tcm16-140203.pdf”'.

[12] Mohit Yadav, Kartik Kanodia, Kusum Grewal Dangi, Dr. Supriya P Panda, "A Survey of Bluetooth and ZigBee WPAN Standards", () 2016 IJEDR | Volume 4, Issue 2 | ISSN: 2321-9939.

[13] Mohit Yadav, Kusum Grewal Dangi, "Cellular Generations: Comparative Analysis ",International Journal of Modern Embedded System (IJMES), ISSN: 2320-9003 (Online), Volume No.-4, Issue No.5, October, 2016.

[14] Neshat Karim Shaukat, "Wi-Fi Direct in Android Using Peer to Peer Communication", International journal for research in applied and engineering technology (ijraset), Vol. 2 Issue I, January 2014 ISSN: 2321-9653.

[15] RANKED Health [Internet] place unknown: publisher unknown; [cited at 2016 Jul 1]. Available from:http://www.rankedhealth.com/about/.

[16] Hackl WO. Intelligent re-use of nursing routine data: opportunities and challenges. Stud Health Technol Inform.2016 225:727-728. [PubMed].

[17] Gazis, V.; Gortz, M.; Huber, M.; Leonardi, A.; Mathioudakis, K.; Wiesmaier, A.; Zeiger, F., Vasilomanolakis, E. (2015), “A Survey of technology for the internet of things" Wireless Communications and Mobile Computing Conference (IWCMC), 2015 International, vol., no., pp.1090-1095, 24-28 Aug. 2015 doi: 10.1109/IWCMC.2015.7289234.

[18] Internet link, http://searchcrm.techtarget.com/definition/real-timeanalytics. 